\title{
Immersiveness and Symmetry in Copresent Scenarios
}

\author{
Ilona Heldal ${ }^{* 1}$, Ralph Schroeder ${ }^{2}$, Anthony Steed $^{3}$, Ann-Sofie Axelsson $^{1}$, Maria Spante $^{1}$, \\ Josef Wideström ${ }^{4}$ \\ ${ }^{1}$ Dept. of Technology and Society, Chalmers University of Technology \\ ${ }^{2}$ Oxford Internet Institute, Oxford University \\ ${ }^{3}$ Dept. of Computer Science, University College London \\ ${ }^{4}$ Digital Media and Communication, Chalmers University of Technology
}

\begin{abstract}
Collaboration at a distance has long been a research goal of distributed virtual environments. A number of recent technologies, including immersive projection technology systems (IPTs) and head-mounted displays (HMDs), promise a new generation of technologies that are more intuitive to use than desktop-based systems.

This paper presents an experiment that compares collaboration in five different settings. Pairs collaborated on the same puzzle-solving task using one of: an IPT connected to another IPT, an IPT connected to an HMD, an IPT connected to a desktop system, two connected desktop systems, or face-to-face collaboration with real objects.

The findings demonstrate the benefits of using immersive technologies, and show the advantages of using symmetrical settings for better performance. Some usability problems of the different distributed settings are addressed, as well as factors such as "presence" and "copresence" and how these contribute to the participants' overall experiences.

CR Categories: H.5.3 [Group and Organization Interfaces]: Computer-supported cooperative work; I.3.7 [Three-Dimensional Graphics and Realism]: Virtual Reality

Keywords: virtual environments, immersive, asymmetry, presence, performance, usability, collaboration.
\end{abstract}

\section{INTRODUCTION}

Collaborative virtual environments (CVEs) aim to support collaborative work or play amongst distributed users. Online game playing is an obvious example. However there have been only few studies examining different types of immersive and non-immersive interfaces to CVEs in networked scenarios $[1,2]$. No previous studies have made extensive comparisons of immersive and non-immersive systems. In this paper, a collaborative task is undertaken with four different combinations of immersive and non-immersive systems. The results are compared to a similar task for faceto-face collaboration with real objects.

It may seem self-evident that asymmetrical settings should be less effective for task performance than settings with the same technology at both ends of the collaboration.

\footnotetext{
*Ilona Heldal, ilohel@mot.chalmers.se
}

However, little is known about the impact of differences between the networked systems on collaboration and on the collaborators themselves. This knowledge would be beneficial since everyday settings are usually asymmetric in some way. Using two different systems in a networked condition does not necessarily mean that the benefits or the drawbacks of the individual systems will characterize the networked setting [3, 4]. There is little knowledge about advantages and drawbacks of using different kinds of distributed settings. Even though immersive systems already have shown their benefits for certain cases $[5,6]$, there are only a few comparative evaluations of how they work when connected to other systems [3].

We will present five different technical settings. One could discuss whether the settings that will be presented here are representative. It would be possible to add various other technologies for comparison, such as holobenches and PowerWalls. A first step, though, is to compare some widely used technologies with some new ones. Given that objectfocused work is quite common [7] we are led to examine how this kind of collaborative work can be supported by using CVEs. We have therefore compared desktop systems with immersive technologies, such as the CAVETM, originally described in $[8]^{1}$ and head-mounted displays (HMDs) for a relatively easy object-focused task.

This paper examines how two people collaborate on a Rubik's cube-type puzzle. In order to solve the puzzle within the given time ( 20 minutes), active interaction with the objects and with the partners was required. The data were collected from 220 subjects ( 22 pairs for each setting).

\section{MOTIVATION}

There are significant differences between interactions in desktop systems and IPT systems. The key difference is that with an IPT system, tracking of the human body contributes to making the interaction more intuitive because actual body posture and gesture are conveyed immediately, rather than having to be expressed through a user interface. We also examined face-to-face collaboration with real objects in order to compare this with how natural the interaction is in the networked settings.

Many issues such as presence, performance, intuitiveness, and leadership have been identified as important in virtual

\footnotetext{
${ }^{1} \mathrm{CAVE}^{\mathrm{TM}}$ is a trademark of the University of Illinois at Chicago. By using the abbreviation IPT (Immersive Projection Technology) we refer to this type of technology, originally designed by Cruz-Neira, Sandin and DeFanti [8].
} 
Table 1. Settings and abbreviations used.

\begin{tabular}{|c|c|}
\hline The Five Settings & Abbreviations \\
\hline $\begin{array}{c}\text { Gothenburg IPT connected to } \\
\text { London IPT }\end{array}$ & I $^{1}-$ I \\
\hline IPT connected to HMD & I - HMD \\
\hline IPT connected to Desktop system & I - D \\
\hline Two connected Desktop systems & D - D \\
\hline Cardboard blocks & Real \\
\hline
\end{tabular}

${ }^{1}$ Notation: The $I$ always refers to the IPT system in Gothenburg when is positioned to the left.

environments $[9,10]$. Certain features such as presence, performance, and immersion may be more closely associated with one specific type of VE than with another [11-13]. For example, desktop systems can be more effective than immersive environments for problem-solving when visualizing large molecules [14] and for educational use [15]; where immersive systems can be more effective for visual modelling [5], and abstract information visualization [16]. There are also differences when varying some technical factors of an environment. For example, varying latency [17, $18]$ or rendering $[19,20]$ results in different performance and presence measures, and using different type of technical interaction possibilities affects usability [21] and the use of various perspectives for collaborative navigation results in differences regarding performance [22]. While the number of CVE applications is increasing, the majority of the studies focus on technical aspects for single-user environments [23], even though social interaction influences experiences and performance in CVEs [3].

\section{Previous studies}

The experiment presented in this study is based on earlier studies. Partial results on performance and presence in the II, I - D and Real settings have been reported previously [24, 25] (see Table 1 for abbreviations used). The first study reported unequal collaboration between the participants involved in the different settings ( $\mathrm{I}-\mathrm{D})$, despite being unaware of what type of system the other participant was using. The second study noted that performance in a networked IPT (I-I) setting was almost as good as in a realworld scenario, especially compared to an I-D setting.

[1]

\section{STUDY DESIGN}

We added a D - D setting because it adds another symmetrical setting and also because it is most common CVE setting. We also added an I - HMD setting.

Although HMDs are immersive like IPTs, they have some qualitative differences such as the user not being able to see their own body. We have not had occasion to examine the HMD-HMD setting to date.
The participants were asked to fill out a questionnaire on their background before the trial and on their experiences after the trial $[24,25]$. We also used observation sheets filled out by an observer for each participant for the virtual settings and for the two participants in the face-to-face scenario.

\subsection{The Puzzle-solving Task}

The puzzle involved 8 separate blocks with different colours on different sides. The pairs had to rearrange the blocks so that each side would display a single colour, i.e. 4 squares of the same colour on each of the six sides (see Figure 1). Therefore, the task was similar to, but less complex than, the popular Rubik's cube puzzle that involves 27 blocks with 9 squares on each side. The squares were $30 \mathrm{~cm}$ along each edge both in reality and in their representations in the VEs. For the Real trial, participants were asked to do the task with cardboard blocks having the same size and colours as the blocks that were represented in the VEs.
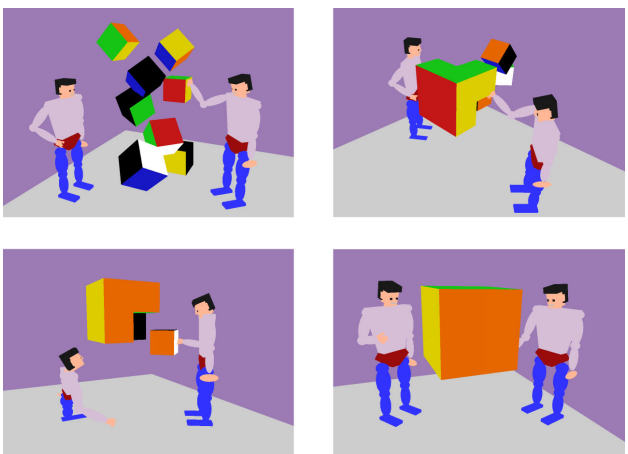

Figure 1. Two participants completing the 3D cube puzzle.

In order to solve the puzzle, the partners needed to actively interact with the objects and each other. They had to move around, turn, examine, and relocate objects. They made full use of the IPT's and HMD's tracking system by bending down and moving around in the space in order to get different viewpoints. The lower left picture in Figure 1 shows a participant bending down to see the colours from below while her/his full-sized partner inserts the last block in its place.

Participants were given a maximum of 20 minutes to solve the puzzle. For the virtual settings they had a short introduction time (between 5 and 10 minutes) until they could mark and pick up objects, navigate around in the environment and use the devices, and use the audio. The participants in the face-to-face condition did not have this introductory timeslot.

There were 220 voluntary participants in the trials and all experienced the system and the task for the first time. They were paired randomly. A participant did not know about her/his partner, and for the virtual settings s/he did not know what technology her/his partner was using.

Each participant was portrayed to the other by the use of a simple avatar with a jointed arm. In the immersive VEs the participant could not see her/his own avatar (virtual 
representation), except for a virtual hand drawn in the same position as the physical hand.

\subsection{Technical Description}

To allow smooth collaboration and create settings that were as equal as possible for the purpose of comparison, adjustments were made so that the environments and the avatars were similar in appearance and functionality. Although local tracker updates are applied at the fastest rate provided by the tracker driver, updates to the remote avatar are only sent at $10 \mathrm{~Hz}$. The network lag between the two sites for the I - I trials was approximately $180 \mathrm{~ms}$, and faster for the I-HMD, I-D, and D - D trial (which took place on the local network at Chalmers University of Technology).

In the immersive systems used, the participants could grab the blocks or cubes by putting their hand into the virtual cube and pressing on the button of the 3-D wand in the case of the IPTs, and by pinching together their thumb and index finger using the glove for the HMD. On the desktop system, participants could navigate by moving the middle mouse button and could select the cubes by clicking on a cube with the left mouse button. To move the cubes, they had to keep the right mouse button pressed and move the mouse in the desired direction. They could also rotate the cubes by pressing the right mouse button combined with the shift key.

The subjects could talk to and hear each other using a wired headset with microphone as well as earphones. We used the Robust Audio Toolkit (RAT) for audio communication between the participants (except when RAT occasionally did not work and had to be replaced by mobile phones).

The Real setting: For the real trial, participants were asked to do the task around a table with lightweight cardboard blocks having the same size and colours as they were represented with in the VEs.

I - I setting: The IPT system at Chalmers was a five-sided (no ceiling) $3 \mathrm{~m} \times 3 \mathrm{~m} \times 3 \mathrm{~m}$ TAN VR-CUBE (henceforth Chalmers Cube). The application was run on a Silicon Graphics Onyx2 Infinity Reality with $14250 \mathrm{MHz}$ R10000 MIPS processors, 2GB RAM and 3 graphics pipes. The participants wore CrystalEyes shutter glasses. A Polhemus magnetic tracking device tracked both the glasses and the wand. The rendering performance was at least $30 \mathrm{~Hz}$. Locomotion was disabled at the Chalmers Cube since the puzzle fits within the space of the Cube.

The IPT system at University College London was a foursided Trimension ReaCTor (henceforth UCL ReaCTor) with a floor of $2.8 \mathrm{~m} \times 2.8 \mathrm{~m}$ and three $2.8 \mathrm{~m} \times 2.2 \mathrm{~m}$ walls. It was powered by a Silicon Graphics Onyx 2 with eight $300 \mathrm{MHz}$ R12000 MIPS processors, 8GB ram and 4 Infinite Reality2 graphics pipes. The participants wore CrystalEyes stereo glasses. The head and wand were tracked by an Intersense IS900 system. The participant used a joystick with 4 buttons. Rendering performance was at least $45 \mathrm{~Hz}$. Since the UCL ReaCTor only has 3 walls plus a floor, it was necessary to enable locomotion with the joystick.
For the I - I condition, the applications were implemented in a customized version of the Distributed Interactive Virtual Environment (DIVE) system [19, 26]. DIVE supports collaborative environments by way of a partially replicated shared database that is maintained by a multicast event protocol. DIVE has an abstract model of interaction that allows a wide range of locomotion and manipulation to be implemented. It is designed to support distribution of environments to mid-sized groups of participants over a wide area. However, given the lack of multicast connectivity between the two sites, we set up a DIVEBONE connection to act as a bridge.

I - HMD setting: The IPT system used in this case was the same as the IPT system at Chalmers in the I - I setting.

The HMD was an $n$-Vision Visor, Datavisor 10x. The field-of-view (FOV) was 50 degrees per eye, with 25\% stereo overlap. Total FOV was Horizontal: $87^{\circ}$, Vertical: $50^{\circ}$. The resolution VGA for each eye was $640 \times 480$ pixels. The locomotion was disabled. The input device used was a Fakespace PINCH ${ }^{\mathrm{TM}}$ Glove.

The software used here was PTC DIVISION Mockup with approximately $60 \mathrm{~Hz}$.

I - D setting: The IPT system in this case was the Chalmers system. The desktop system comprised a Silicon Graphics O2 with one MIPS R10000 processor and 256MB RAM and a 19-inch screen display. The rendering performance was at least $20 \mathrm{~Hz}$. An ordinary mouse was used for navigation on the desktop systems. We used dVise 6.0 software for the distributed application.

D - D setting: We used the same Desktop-type systems for this setting as the Desktop system described above. The software used here was DIVISION Mockup.

\section{RESULTS}

\subsection{Performance}

We measured the time each pair took to solve the task. Pairs that did not complete the task within the time limit of 20 minutes were interrupted and not given a specific time measure.

Figure 2 shows the cumulative results for each setting. $50 \%$ of the subjects who solved the task in the Real setting solved it in just under 8 minutes. The same percentage solved the task in the I - I in just above 8 minutes, for I - HMD in around 19 minutes and for $\mathrm{D}-\mathrm{D}$ in just under 20 minutes. Thus we have the expected rank order Real, I - I then I HMD. However, the number of groups who completed the task in the D - D setting was high and, contrary to prior expectations, higher than for the I $-\mathrm{D}$ setting. 


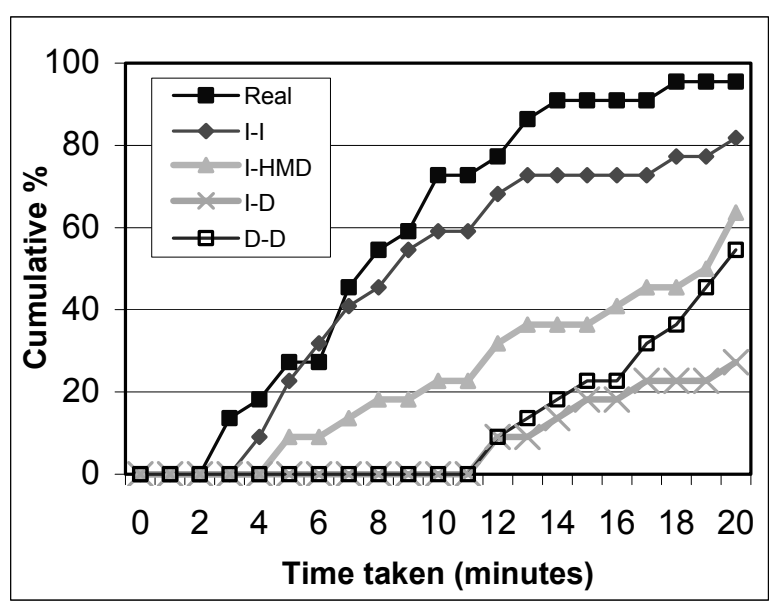

Figure 2.Cumulative percentage of pairs that completed the task in the given time.

It is worth observing in Figure 2 shows that the curves for Real and I - I setting are quite similar, with a few groups completing the task very quickly indeed. This suggesst that that the learning threshold for the IPT is low. However, contrast this with $\mathrm{I}-\mathrm{D}$ and $\mathrm{D}-\mathrm{D}$, where no group finishes until 12 minutes have passed. Of the 110 groups, 71 managed to solve the puzzle.

\subsection{Collaboration}

It was important to see if individual participants estimated that they took an equal part in collaboration. To indicate her/his level of collaboration each participant responded to three questions: "To what extent did you experience that you and your partner collaborated?" "Think of some previous time (before today) when you enjoyed collaborating with someone. To what extent did you enjoy collaborating with your partner in today's task?" "To what extent would you, on another occasion, like to carry out a similar task with your partner?". ANOVA was used to find statistical significance.

There was no significant difference $(p>.05)$ across the five different conditions for any of the questions. The participants marked responses on a scale of $1-5$, where 1 was to a very small extent and 5 was to a very large extent. The mean values for all three questions and for all settings were in the interval of $3.9 \pm 0.4$ (with SD between $0.7 \pm 0.3$ ). The relatively high values show that people on average enjoyed collaboration. There was no significant difference between the different settings even when we only considered the pairs that managed to solve the task.

\subsection{Contribution to the Task}

To estimate individual contributions to the task we asked each participant to estimate her/his own contribution. Evaluations were given in percentages where both partners had to add up to $100 \%$; i.e. if they acted equally the responses would be 50 and 50 . The results for all settings show that pairs estimated the contributions realistically. Here we must note again that the people answering these questions had no knowledge of the system their partners were using nor about their partners and her/his answers.

To find out the contributions of the partners in solving the task they estimated their own (and the partner's) contribution to (T1) solving the tasks in general, (T2) placing the cubes, and (T3) how much they were talking during problem solving.

The results from ANOVA showed no significant differences for verbal communication for all five settings. Significant differences were found for the asymmetrical I HMD and also for the I - D settings for contribution to solving the task and to placing the cubes. The most significant differences $(p<0.002)$ were found for the I HMD setting. For this condition, the participant working with the HMD estimated her/his own collaboration as much lower $(\mathrm{M}=28.23, \mathrm{SD}=19.90$ for own contributions to solving the task, and $\mathrm{M}=28.86, \mathrm{SD}=18.64$ for placing the cubes) than that of the partner in the IPT system $(\mathrm{M}=70.91$ and $\mathrm{SD}=18.87$, respectively $\mathrm{M}=76.82$ and $\mathrm{SD}=18.67)$.

\subsection{Presence}

Presence is an important factor in the experience of an environment. It can be defined as the sense of being in a computer-generated place other than that in which one is physically present [27]. Presence was gauged by the method previously used by Slater and colleagues [2].

We asked about presence on two occasions (for control) and by asking two differently formulated questions: "To what extent did you have the same experience that you where in the same room as the cubes?" and "When you think back on the task, to what extent can you have the experience right now that you are moving around in the same room where the cubes were?" There were no significant differences between the answers given for the differently formulated questions.

The only significant differences between the mean values were found for the I - D setting (see Figure 3). The answers were values on a scale of $1-5$ where $1=$ to a very small extent and $5=$ to a very high extent.

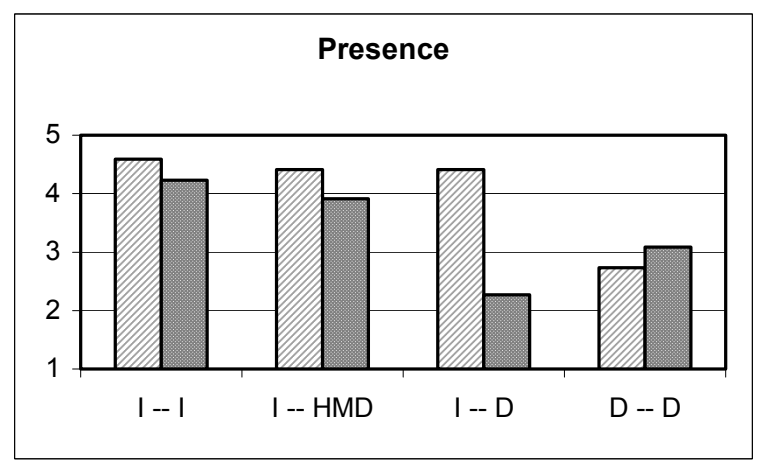

Figure 3. Estimated presence in the four CVEs.

We used the same type of desktop system in the D - D setting, whereas I - I might reflect an actual difference due to the different displays. 
Presence for all three immersive environments was higher. Participants experienced a lesser level of presence if they worked on a Desktop system, regardless of whether this was connected to an immersive system or to another Desktop system. Note that the results for the desktop system in the ID setting was lower than either desktop systems in the D-D setting.

\subsection{Copresence}

If presence is defined as the sense of being in the computergenerated environment, copresence is the subjective sense of being there together and interacting with each other [28]. In order to find out about the level of copresence (we used the same scale of $1-5$, where $1=$ to a very small extent and $5=$ to a very high extent), we again asked two differently formulated questions: "To what extent did you have the sense that you were in the same room as your partner?" "When you continue to think back on the task, to what extent can you have a sense that you are together with your partner in the same room?"

There were no significant differences between the answers for the two differently formulated questions. This indicates that the participants interpreted copresence in the same way on both occasions.

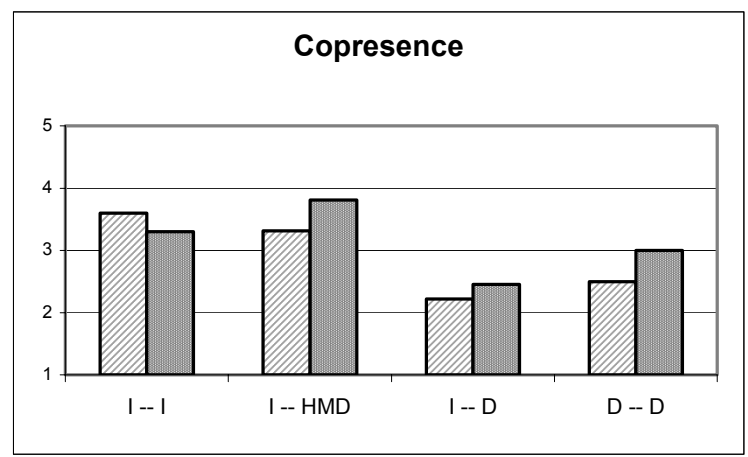

Figure 4. Estimated copresence in the four CVEs.

The pairs in the two immersive settings reported a higher sense of copresence than the pairs in the I - D and D - D settings. The copresence reported by the participants working in the HMD was highest (see Figure 4).

It is interesting to note the inverse relation between presence and copresence for the I - HMD and I - D settings (see Figure 3 and Figure 4).

\subsection{Usability issues}

Earlier we presented collaborative aspects of usability, i.e. performance and contribution to the task. Here we concentrate on single user characteristics that contribute to effectiveness and efficiency, viz. manipulation and ease to see objects.

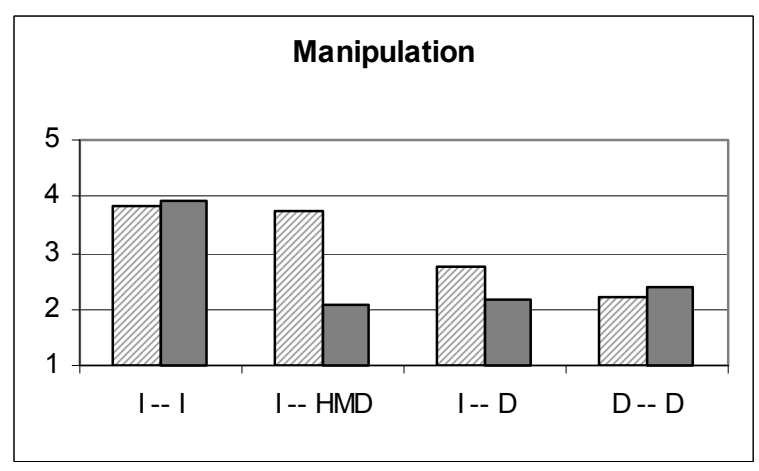

Figure 5. Ease of manipulation in the four CVEs.

The easiest manipulation occurred in the IPT systems. Using the HMD and Desktop systems, the participants found manipulation difficult.

In the I - D setting, the participant using the IPT system found manipulation almost as difficult as the participant using the Desktop systems (see Figure 5). The answers to the question: "To what extent could you move and handle the cubes as you wanted to?", were again on a scale from 1 to 5 with $1=$ very hard to manipulate and $5=$ very easy.

There were significant differences between the systems if they were connected to a different type of system. While in the Chalmers IPT system, when this was connected to immersive systems, the participants thought that they could manipulate the objects easily $(\mathrm{M}=3.82, \mathrm{SD}=0.09$ for $\mathrm{I}-\mathrm{I}$, and $\mathrm{M}=3.73, \mathrm{SD}=1.16$ for $\mathrm{I}-\mathrm{HMD}$ ), the manipulation was considered to be harder when the Chalmers IPT was connected to a Desktop system $(\mathrm{M}=2.77, \mathrm{SD}=0.68$ for the $\mathrm{I}-$ D setting).

The participants working on a Desktop or HMD reported several problems regarding manipulation and handling the objects in the environment. This could be a result of the short period of time getting used to these systems (30 minutes if we include training time) may be too short compared with the IPT systems.

The answers to the question regarding "How easy or difficult was it to see the different sizes of the cubes and how they fitted together?" were quite similar to those for manipulation.

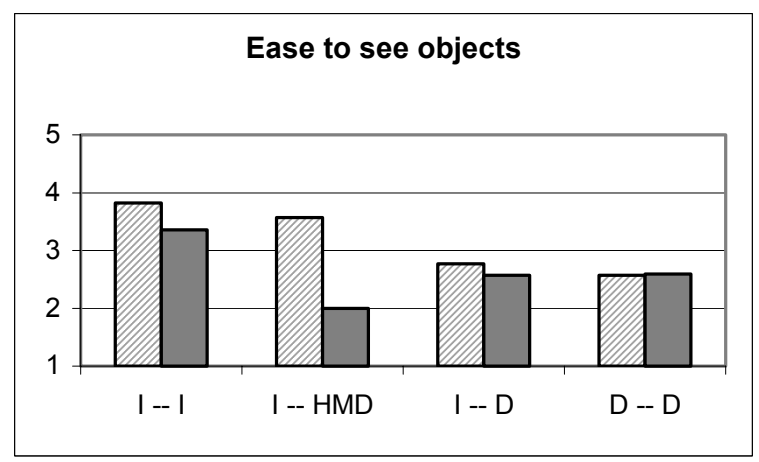

Figure 6. The ease of seeing objects and how they fitted together. 
Here it has to be noted that on the rating of the ease to see objects, the desktop system in both the D - D and the I-D settings scored higher than the HMD in the I - HMD setting. The benefits of the desktop system for seeing the objects, together with the difficulties to move them may also suggest that what is needed for less intuitive systems, is more support for learning to handle the respective applications.

The relatively low value for the HMD system for manipulation and also for the ease to see objects might be due the used HMD's limited field of view.

\section{Discussion}

This research was aimed at understanding the impact of using different types of distributed graphical environment. By having two different immersive systems and four different networked settings for object-focused collaboration, we are able to provide an account of how the systems, including asymmetrical settings can be related to each other for object focused interaction.

Performance was best in the immersive virtual environments. There were no significant differences between the immersive systems involved in this study. However, the performance, experience of presence and co-presence, manipulation and fitting the objects together was better for both IPT systems than the HMD system used. The differences are not great, which leads us to hypothesize that subtle differences might lead to asymmetry in the collaboration. A recent study has shown that IPTs afford faster performance on base interaction tasks such as selection and manipulation [29]. Another assumption that could be discussed whether the relatively poor performance results of I - HMD depends on the asymmetry or simply on the performance of the HMD system used in this experiment.

The poor performance for the $\mathrm{I}-\mathrm{D}$ setting in relation to I - HMD points to the fact that much depends on unequal collaboration. Since the results for the D - D setting are surprisingly close to the I - HMD and I - D results, equal collaboration must be a key factor.

Even if the collaborators do not know much about the system that their partners are using, when they become familiar with the differences, they collaborate accordingly. When they become aware of the advantages and disadvantages of the partner's system, they exploit it, e.g. for the I - D asymmetrical collaboration the person with a desktop system often asks the partner in the IPT system to move the virtual objects [4] since she or he obviously finds it easier to perform the requisite actions. By knowing from the beginning what kind of system the partners are using, and experiencing that system as well, was also shown to be beneficial for I - D asymmetric settings [30].

There were differences between the two IPT systems as well. Working in the IPT system at Chalmers allowed a greater sense of presence, but lower values for manipulation and for fitting the objects together than the IPT system at UCL. This could be a result of differences between the more surrounding system (with 5 walls) and more clumsy devices at Chalmers and the less surrounding system (4 walls) and more accurate tracking devices at UCL.
A previous study has identified some usability guidelines for CVEs [3], but it does not examine different systems and the differences in collaborating in symmetric and asymmetric networked settings. One practical implication is that for object-focused problem solving upgrading one of two nonimmersive systems to an immersive system is sometimes counterproductive.

The estimations for overall collaboration in the questionnaires do not show great differences between the different settings, which is consistent with our previous results [25]. This demonstrates that the applied quantitative methods for studying differences between successful and failing collaboration, are not enough. A possible future investigation is therefore to examine collaboration by using qualitative analysis and examining the video- and audio recordings. The first direct investigation may be to further analyze responses to the open-ended questions for the couples who collaborated effectively and for those who failed.

In our future research, we plan to carry out the single user trials with the real cardboard blocks and for the different VEs, to estimate the added value of collaboration. Other trials we need to include are those that use an HMD with better technical performances, and possibly two HMDs in a networked setting. It is clear that further research is needed to examine different other tasks, and explore other forms of collaboration, since this may produce somewhat different results.

Comparing different networked setting implies also the need for more empirical studies and also more theoretically informed studies on the use of networked scenarios. Further systematic comparisons between symmetrical and asymmetrical settings will yield valuable lessons for usability and point the way towards formulating more refined questions for the CVEs of the future.

\section{CONCLUSIONS}

This study compared four different distributed settings of virtual environments for collaborating pairs solving an object-focused task. The findings were compared to a faceto-face collaboration with real objects doing the same task. In terms of the number of groups who managed to complete the task and their time to solve it, the results demonstrated that people working in distributed immersive environments felt more present and were more effective than those in nonimmersive settings.

The performance results for the best immersive setting were close to the performance results for working together in a face-to-face setting and with real objects. Together with the finding that copresence was significantly higher for the two immersive settings compared with the settings where no immersive systems were involved, this motivates us to study efficient collaboration for different immersive settings. Even if there were difficulties with asymmetry (equal contribution to task, manipulation and ease to see objects), the subjects working in the I - HMD setting performed better than those in the D $-\mathrm{D}$ setting. This shows that immersiveness might contribute substantially to object focused problem solving. 
For asymmetrical settings (e.g. connecting an immersive system to a non-immersive one, and also within the immersive settings, connecting the IPT to the HMD) the benefits of using immersive systems are lower. The main problems here are: lower copresence, manipulation difficulties, and problems in seeing the objects and how they fit together. Contribution to solve the task and performance were also lower.

These aspects are most evident for the immersive system when connected to the non-immersive setting. In this case the cumulative number of groups who completed the tasks and the level of reported copresence during the trial were significantly lower than for the networked desktop setting.

These findings demonstrate the practical advantages of using symmetrical settings even if they are non-immersive ones.

\section{ACKNOWLEDGEMENTS}

Thanks to Lars Bråthe, from Volvo Powertrain for his advices, and to David Swapp for supporting the IPT trials at UCL. This work was supported in part by the UK Equator project (EPSRC Grant GR/N15986/01).

\section{REFERENCES}

[1] Leigh, J., Johnson, A., et al., 1999, Visualization in Teleimmersive Environments, IEEE Computer, December, p. 66-73.

[2] Slater, M., Sadagic, A., et al., 2000, Small-group behavior in a virtual and real environment: A comparative study, Presence: Teleoperators and Virtual Environments, 9(1), p. 37-51.

[3] Tromp, J., Steed, A., et al., 2003, Systematic Usability Evaluation and Design Issues for Collaborative Virtual Environments, Presence: Teleoperators and Virtual Environments, 10(3), p. 241-267.

[4] Heldal, I., 2003, The Treatment of Collaboration in the Usability Evaluation Models for Collaborative Virtual Environments, Proc. 10th International Conference on HCI, p. 914-918.

[5] Neubauer, B. J. and Harris, J. D., 2003, Immersive Visual Modeling: Potential Use of Virtual Reality in Teaching Software Design, The Journal of Computing in Small Colleges, 18(6), p. 142-150.

[6] Slater, M., V. Linakis, et al., 1996, Immersion, Presence, and Performance in Virtual Environments: An Experiment with Tri-Dimensional Chess, Proc. ACM Virtual Reality Software and Technology, p. 163-172.

[7] Hindmarsh, J., Fraser, M., et al., 2000, Virtually Missing the Point: Configuring CVEs for Object-Focused Interaction, In Churchill, E.F., Snowdon, D.N., et al. eds. Collaborative Virtual Environments, Springer: London, p. 115-142.

[8] Cruz-Neira, C., Sandin, D., et al., 1993, SurroundScreen Projection-Based Virtual Reality: The Design and Implementation of the CAVE, Proc. Proceedings of SIGGRAPH 93, p. 135-142.
[9] Steed, A., Slater, M., et al., 1999, Leadership and collaboration in virtual environments, Proc. IEEE Virtual Reality, p. 112-115.

[10] Draper, J. V., Kaber, D. B., et al., 1998, Telepresence, Human Factors, 40(3), p. 354-375.

[11] Slater M., V. Linakis, et al., 1996, Immersion, Presence, and Performance in Virtual Environments: An Experiment with Tri-Dimensional Chess, Proc. ACM Virtual Reality Software and Technology, p. 163-172.

[12] Sallnäs, E. L., 2002, Collaboration in Multi-modal Virtual Worlds: Comparing Touch, Text and Voice and Video, In Schroeder, R. ed. The Social Life of Avatars Presence and Interaction in Shared Virtual Environments, Springer: London, p. 172-187.

[13] Bystrom, K. E. and Barfield, W., 1999, Collaborative Task Performance for Learning Using a Virtual Environment, Presence: Teleoperators and Virtual Environments, 8(4), p. 435-48.

[14] Heldal, I. and Schroeder, R., 2002, Performance and Collaboration in Virtual Environments for Visualizing Large Complex Models: Comparing Immersive and Desktop Systems, Proc. 8th International Conference on Virtual Systems and Multimedia, VSMM2002, p. 208-220.

[15] Youngblut, C., 1998, Educational Uses of Virtual Reality Technology. Report, Institute for Defense Analyses 1801 N. Beauregard St. Alexandria, VA 22311-1772, p. 123.

[16] Bowman, D. A. and Raja, D., 2004, A Method for Quantifying the Benefits of Immersion Using the CAVE, Presence-Connect, 4(4).

[17] Arthur, K. W. and Booth, K. S., 1993, Evaluating 3D Task Performance for Fish Tank Virtual Worlds, $A C M$ Transactions on Information Systems, 11(3), p. 239265.

[18] Meehan, M., Razzaque, S., et al., 2003, Effect of Latency on Presence in Stressful Virtual Environments, Proc. IEEE Virtual Reality Conference 2003 (VR 2003), p. 141-148.

[19] Steed, A., Mortensen, J., et al., 2001 Spelunking: Experiences using the DIVE system on CAVE-like platforms, In Frohlicj B.,Deisinger, J. and Bullinger, H. eds., Immersive Projection Technologies and Virtual Environments, Springer-Verlag: Wien, p. 153-164.

[20] Zimmons, P. and Penter, A., 2003, The Influence of Rendering Quality on Presence And Task Performance in a Virtual Environment, Proc. IEEE Virtual Reality Conference 2003 (VR 2003).

[21] Bowman, D., Gabbard, J., et al., 2002, A Survey of Usability Evaluation in Virtual Environments: Classification and Comparison of Methods, Presence: Teleoperators and Virtual Environments, 11(4), p. 404424.

[22] Yang, H. and Olson, G. M., 2002, Exploring Collaborative Navigation: the Effect of Perspectives on Group Performance, Proc. CVE 2002, p. 137-142.

[23] Wilson, J. R., 2003, If VR has changed. then have its human factors? In Waard, D.d., Brookhuis, K.A., et al. 
eds. Human Factors in the Age of Virtual Reality, Shaker Publishing: Maastrich, p. 9-30.

[24] Wideström, J., Axelsson, A., et al., 2000, The Collaborative Cube Puzzle: A Comparison of Virtual and Real Environments, Proc. CVE 2000, p. 165-171

[25] Schroeder, R., Steed, A., et al., 2001, Collaborating in Networked Immersive Spaces: as Good as Being there Together? Computers \& Graphics, 25(5), p. 781-788.

[26] Frecon, E., Smith, G., et al., 2001, An overview of the COVEN platform, Presence: Teleoperators and Virtual Environments, 10(1), p. 109-127.

[27] Slater, M. and Steed, A., 2000, A Virtual Presence Counter, Presence: Teleoperators and Virtual Environments, 9(5), p. 413-434.
[28] Schroeder, R., 2002, Copresence and Interaction in Virtual environments: An overview of the Range of Issues, Proc. Fifth International Workshop Presence 2002, p. 274-95.

[29] Steed, A. and C. Parker, 2004, Evaluating Effectiveness of Interaction Techniques across Immersive Virtual Environment Systems, Forthcoming in Presence: Teleoperators and Virtual Environments.

[30] Spante, M., Schroeder, R., et al., 2004, The Good Inequality: How the Experience of Different CVE Technologies Enhances Collaboration, In Schroeder, R. and Axelsson, A.-S. eds. Forthcoming: Avatars at Work and Play: Interaction and Collaboration in Shared Virtual Environments: London: Springer. 